In der Rubrik „Literatur kompakt" werden die wichtigsten Originalarbeiten aus der internationalen Fachliteratur referiert.

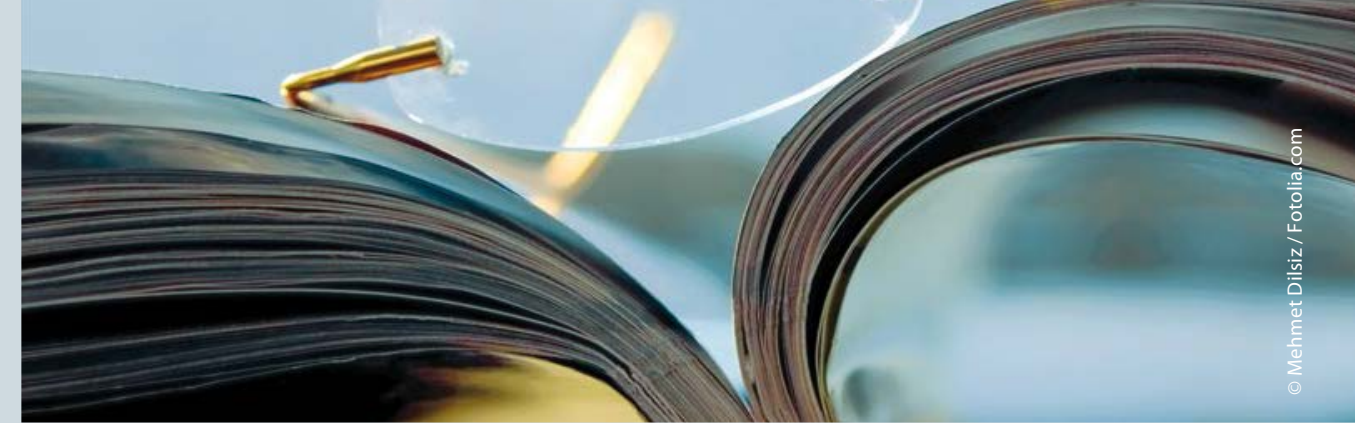

\section{Placebovergleich bei Ischialgie: Orale Steroide wirken nur schwach}

\author{
Der Einsatz von oralen Steroiden bei akuter Radikulopathie infolge eines \\ lumbalen Bandscheibenvorfalls bessert allenfalls die Funktion, aber nicht \\ den Schmerz.
}

G emäß der S2-Leitlinie zur lumbalen Radikulopathie kann „die orale Kortikoidgabe in einer Dosis von 50$100 \mathrm{mg}$ Prednisolon pro Tag empirisch insbesondere bei foraminalen Hernien kurzfristig zu einer deutlichen Schmerzreduktion und auch Funktionsverbesserung führen“. Einschränkend wird allerdings darauf hingewiesen, dass „suffiziente Studien, die diesen Eindruck belegen, nicht vorliegen“.

Der US-amerikanische Gesundheitsdienstleister Kaiser Permanente Northern California hat nun eine solche Studie nachgereicht. Und deren Ergebnisse können diesen Eindruck keineswegs belegen. „Die Kurzzeittherapie mit einem oralen Steroid bewirkte eine mäßige Verbesserung der Funktion und keine Verbesserung der Schmerzen“, berichten die Harley Goldberg und Kollegen.

An der randomisierten placebokontrollierten Doppelblindstudie hatten sich 269 Patienten beteiligt, die seit höchstens drei Monaten an radikulären Schmerzen litten, einen per

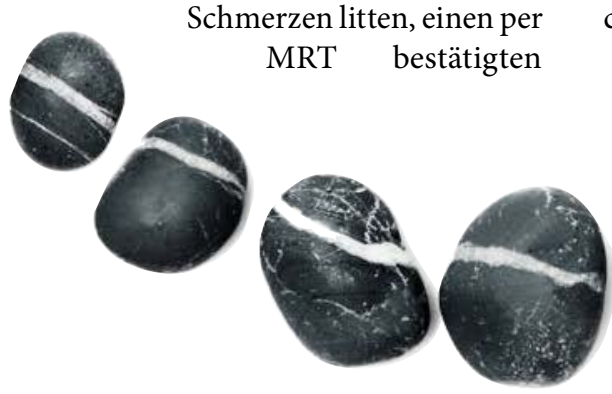

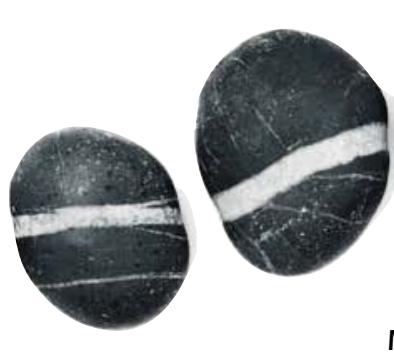

Bandscheibenvorfall hatten und im Oswestry Disability Index (ODI) mindestens 30 von schlimmstenfalls 100 Punkten erreichten. 181 Teilnehmer wurden 15 Tage lang mit absteigenden Dosen Prednison behandelt (jeweils fünf Tage 60, 40 und $20 \mathrm{mg}$ ), 88 erhielten ein Placebo.

Der Funktionsstatus gemäß ODI hatte sich nach drei Wochen in beiden Gruppen signifikant gebessert, und zwar von 51,2 auf 32,3 Punkte unter Prednison und von 51,1 auf 37,5 Punkte unter Placebo. Nach statistischer Bereinigung ergab dies eine um 6,4 Punkte größere Reduktion in der Steroidgruppe. Die Chance auf einen Rückgang des ODIScores um mindestens 30 Punkte wurde durch das Steroid um $70 \%$ erhöht. Um diese Verbesserung bei einem Patienten zu erreichen, müssten rein rechnerisch

Der Summenscore für die physische Komponente des SF-36 hatte sich nach drei Wochen ebenfalls 10,6 Patienten behandelt werden.

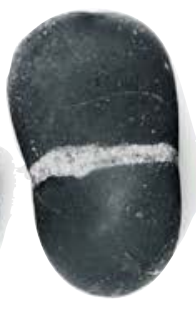

Modell der Wirbelsäule aus Steinen. Akute Schmerzen betreffen oft die LWS. in der Prednisongruppe stärker gebessert. Nach 52 Wochen war hier kein Unterschied mehr zu erkennen.

Keinerlei Einfluss hatte die Steroidtherapie auf die in die Beine ausstrahlenden Schmerzen der Patienten. Sie waren in beiden Gruppen nach drei Wochen und nach einem Jahr gleichermaßen zurückgegangen. Außerdem unterzog sich fast jeder zehnte Patient im Lauf des Jahres einer Wirbelsäulen-Operation - unabhängig davon, ob er Steroide oder das Placebo erhalten hatte.

Fazit: Es bleibt die Frage, ob die mit dem Steroid erzielte Veränderung im ODI von 6,4 Punkten auf einer 100-PunkteSkala eine Behandlung begründen kann. Die minimale Differenz im ODI, die als klinisch relevant angesehen wird, variiert in der Literatur zwischen 5 und 15 Punkten. „Ob die beobachtete Funktionsverbesserung die Therapie mit oralen Steroiden rechtfertigt, ist eine schwierige Entscheidung, die letztlich individuell abgewogen werden muss", resümieren die Autoren. Dr. Beate Schumacher

Goldberg $\mathrm{H}$ et al. Oral Steroids for Acute Radiculopathy Due to a Herniated Lumbar Disk. A Randomized Clinical Trial. JAMA2015; 313(19): 1915-23; doi:10.1001/jama.2015.4468

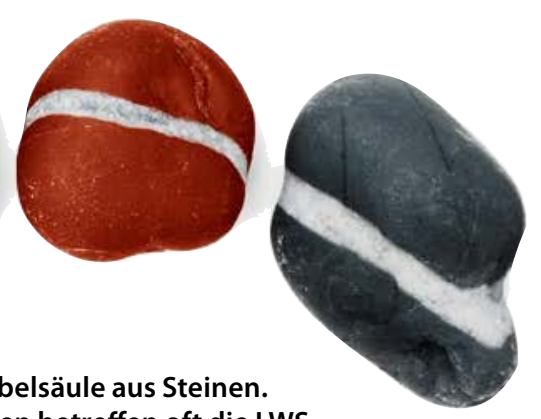

